\title{
Williams Syndrome and Memory: A Neuroanatomic and Cognitive Approach
}

\author{
Adriana Sampaio - Nuno Sousa $\cdot$ Montse Férnandez \\ Cristiana Vasconcelos • Martha E. Shenton • \\ Óscar F. Gonçalves
}

Published online: 26 January 2010

(C) Springer Science+Business Media, LLC 2010

\begin{abstract}
Williams Syndrome (WS) is described as displaying a dissociation within memory systems. As the integrity of hippocampal formation (HF) is determinant for memory performance, we examined HF volumes and its association with memory measures in a group of WS and in a typically development group. A significantly reduced intracranial content was found in WS, despite no differences were observed for HF absolute volumes between groups. When volumes were normalized, left HF was increased in WS. Moreover, a lack of the normal right $>$ left HF asymmetry was observed in WS. No positive correlations were found between volumetric and neurocognitive data in WS. In sum, a relative enlargement of HF and atypical patterns of asymmetry suggest abnormal brain development in WS.
\end{abstract}

Keywords Williams Syndrome - Neurodevelopment . Hippocampal formation - Memory performance

A. Sampaio (凶) · M. Férnandez · Ó. F. Gonçalves

School of Psychology, CIPsi, Neuropsychophysiology Lab,

University of Minho, Braga, Portugal

e-mail: adriana.sampaio@iep.uminho.pt

A. Sampaio $\cdot$ N. Sousa

Life and Health Sciences Research Institute, University of Minho, Braga, Portugal

C. Vasconcelos

Department of Neuroradiology, Hospital Geral Santo António,

Porto, Portugal

M. E. Shenton

Psychiatry Neuroimaging Laboratory, Department of Psychiatry, Brigham and Women's Hospital, Harvard Medical School, Boston, USA

\section{Introduction}

Williams syndrome (WS) is a neurodevelopmental disorder, characterized by a submicroscopic deletion on chromosome 7 q11.23 (Korenberg et al. 2000). Associated with this genotype, WS patients display an unusual cognitive phenotype, characterized by mental retardation with a distinctive neuropsychological profile, with peaks and valleys of abilities. A peak-valley profile has also been proposed within each cognitive domain, with WS memory abilities being characterized by a differential pattern of performance where spared auditory short term memory performance is contrasted with impaired auditory long term memory performance (absence of primacy effect in a word list task in WS group) (Vicari et al. 1996). However, recent studies claim that WS patients manifest global impairment of memory skills, showing that verbal and visual short and long term memory storage systems are equally affected (Brock et al. 2006; Sampaio et al. 2008).

Underlying this asymmetric memory performance, neuroimaging studies have addressed the structural and functional role of Hippocampal Formation (HF) in WS (Chiang et al. 2007; Meyer-Lindenberg et al. 2005; Reiss et al. 2004), a highly specialized brain region involved in memory performance. These studies report HF volume preservation in WS patients with mental retardation (Chiang et al. 2007; Reiss et al. 2004) and in patients with normal IQ, despite evidence of functional abnormalities in this brain region (Meyer-Lindenberg et al. 2005). Thus, one may hypothesize that impairment in memory abilities in WS may be associated with a dysfunctional HF. Of note, however, even though previous studies have acknowledged the importance of HF in understanding the WS cognitive phenotype, no empirical studies to-date have been conducted to explore hippocampus-dependent cognitive 
functions in this syndrome. The $\mathrm{HF}$ is a structure involved in declarative memory, spatial navigation tasks (Eichenbaum et al. 1999; Maguire et al. 2000), working memory (Gazzaley et al. 2004; Karlsgodt et al. 2005; Ranganath and D'Esposito 2001) and long term memory efficiency (Ranganath et al. 2005). The HF is a lateralized structure, showing right $>$ left volume, evident both in children (Giedd et al. 1996; Utsunomiya et al. 1999) and in adults (Pedraza et al. 2004; Pruessner et al. 2000), which may be associated with lateralized functional specialization. In fact, there is evidence to suggest that the processing of verbal memory items is associated with left hippocampus activation while the processing of nonverbal items is associated with right hippocampus activation (Casasanto et al. 2002; Papanicolaou et al. 2002; Piekema et al. 2006).

In this study, we predicted abnormal HF volume and laterality in WS compared with controls. Thus, neuroimaging and neuropsychological evaluations were conducted in a group of WS patients and age-matched controls. We used automated segmentation techniques to evaluate the volume of total intracranial contents, and gray and white matter volume, as well as manual measures to evaluate left and right HF volume and asymmetry in WS patients and controls. We also correlated left and right HF volume with several verbal and nonverbal memory functions.

\section{Method}

Participants

In this study, fifteen participants ( 7 males and 8 females), diagnosed with Williams Syndrome (mean $\pm \mathrm{SD}$ age, $18.53 \pm 5.29$; Full Scale IQ mean: $46.43 \pm 7.45)$ were compared with 13 age-matched healthy controls $(5$ males and 8 females, mean \pm SD age, $19.33 \pm 5.80$; age-range: 11-29 years; Full Scale IQ mean: $110.33 \pm 11.23)$. Participants with WS were recruited at the Genetic Medical Institute (Portugal) and the Genomic Foundation in Galicia (Spain). WS Diagnosis were made by fluorescent in situ hybridization confirmation of elastin gene deletion (Korenberg et al. 2000). Exclusion criteria for typically developing individuals were the evidence of psychiatric, neurological disorder or cognitive impairment. Each participant gave written informed consent for their participation in the study via consent forms, after a complete description of the study. Handedness was controlled (all participants but one were right-handed-determined through clinical observations).
Neurocognitive Assessment

Both groups were assessed using Wechsler Intelligence Scales (3rd Edition)_Children (1991) and Adult (1997) version. In addition, the California Verbal Learning Test (Children and Adult Versions-CVLT) (Delis et al. 1994, 2000) was used for a detailed assessment of verbal memory and learning abilities (the total number of recalled words in 5 trials, long-term memory and recognition variables were used in correlational analyses). Raw scores on the Digit Span subtest from the Wechsler Intelligence Scale and the Corsi Blocks Span (Corsi 1972) were used to assess phonological loop and visuo-spatial working memory, respectively. These tests were used because several studies point out the involvement of medial temporal lobe in the encoding durable memories (Carr et al. in press; Fernandez et al. 1999; Kato et al. 1998). Free recall of visual material was tested using copy and memory conditions of the ReyOsterrieth Complex Figure (Rey 1959), which was scored with a developmental coding system (Bernstein and Waber 1996).

\section{MRI Acquisition and Processing}

MRI images were obtained on a 1.5-T General Electric system (GE Medical Systems). The scan acquisition protocol consisted of contiguous $1.5-\mathrm{mm}$ slices of the whole brain. Coronal T1 (SPGR) acquisition $(256 \times 192$ matrix $)$, $1.5 \mathrm{~mm}$ thickness and an axial PD/T2 sequence were aligned. Images were aligned by using the line between the anterior and posterior commissures and the sagittal sulcus to correct head tilt and were also resampled to make voxels isotropic (sides measured $0.9375 \mathrm{~mm}$ ). Then, an Atlas Based Expectation Maximization (EM) segmentation program separated raw MR data into cerebrospinal fluid (CSF), gray matter (including cortical and cerebellar cortices, basal ganglia and hippocampal-amygdaloid complex) and white matter (Pohl et al. 2004).

\section{Regions of Interest Definition}

Hippocampal formation (HF) was outlined manually using the 3D Slicer Software (http://www.slicer.org/), a free open source software package that allows for three-dimensional visualization of multi-modal image data, segmentation, and advanced image analysis. Hippocampal formation was segmented using the Duvernoy Atlas (Duvernoy 2005) (see Fig. 1) and the Pantel et al. (2000) method, described in detail elsewhere (Pantel et al. 2000). Briefly, after realigned and resampled, we segmented HF in the SPGR images in the coronal plane (with auxiliary guideline traces 

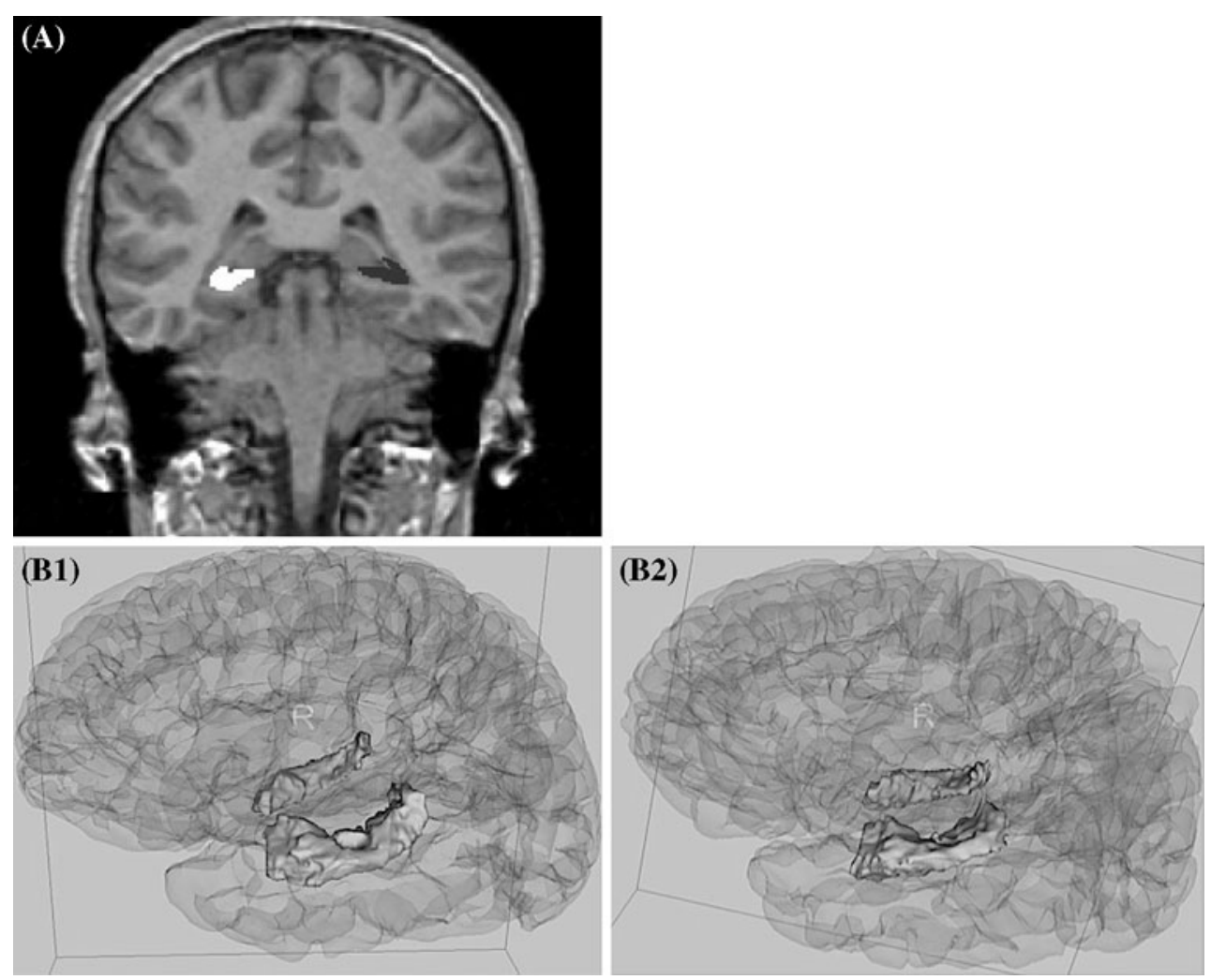

Fig. 1 a Coronal plane of hippocampal formation segmentation: right hippocampal formation (white) and left hippocampal formation (gray); b 3D Reconstruction of HF in WS (B1) and normal control (B2)

in sagital view). The anterior border is outlined by alveus and uncal recess (separating hippocampal head from amygdala; this slice was carefully analysed), laterally, CSF from temporal horn of lateral ventricles limits the body (and in the tail of HF, lateral border is defined by the ascending crus of the fornix and CSF of lateral ventricles); ventral border is defined by the white matter of temporal lobe; the pulvinar thalamus is a limit for the tail of hippocampus. Two raters, blind to group diagnosis, measured the hippocampal formation for all subjects. Inter-rater reliability was $>0.90$ for both structures. The asymmetry index for HF was computed according to the following expression: $(\mathrm{L}-\mathrm{R}) / 0.5(\mathrm{~L}+\mathrm{R})$, where $\mathrm{L}$ and $\mathrm{R}$ refer to absolute volumes in the left and right hemispheres. Adjusted HF formation was computed as the ratio between $\mathrm{HF}$ volume and overall gray matter volume instead of Total Intracranial Content (TIC). The option for this adjustment was due to the following reasons: (1) white matter, gray matter and CSF volumes present differential distribution patterns in the neuroanatomic organization of WS brain that differs from typically development (see (Sampaio et al. 2008); (2) this method of adjustment was previously used in other neurodevelopmental disorders (Pinter et al. 2001); (3) the Atlas Based Expectation Maximization (EM) segmentation program that allow us to classify different brain tissues (white matter, CSF and gray matter), includes in the gray matter tissue classification the hippocampal-amygdaloid complex and; (4) HF was manually segmented as gray matter. Total intracranial content (TIC) was the sum of gray matter, white matter and CSF volumes.

\section{Data Analysis}

Data were analyzed to check for normality and variance homogeneity. All volumetric data, except for white matter volume, met the criteria for the use of parametric tests, including normality (Kolmogorov-Smirnov and ShapiroWilk tests) and variance homogeneity (Levene test). Thus, the non-parametric Mann-Whitney test was used to compare white matter volumes and memory performance between groups. A repeated-measure ANOVA was used to determine HF volume differences between the WS and typically development subjects, where Diagnosis (WS and typically development group) was used as between-subject factor and hemispheric side (left vs right) as the withinsubject factor. Post hoc group differences were performed using a Student's $t$ test. Pearson and Spearman correlations were used in correlational analysis, separately for each group, according to normality and variance homogeneity criteria. Additionally, partial correlations (controlling for 
Table 1 Demographic characteristics

\begin{tabular}{lll}
\hline & $\begin{array}{l}\text { WS group } \\
(N=15) \\
M(\mathrm{SD})\end{array}$ & $\begin{array}{l}\text { Typically developing } \\
\text { group }(N=13) \\
M(\mathrm{SD})\end{array}$ \\
\hline Age & $18.53(5.29)$ & $19.33(5.80)$ \\
FSIQ & $47.43(7.45)$ & $110.33(11.23)$ \\
& $M d n$ & $M d n$ \\
Level of education & 9 & 10 \\
Socio-economic level & 4 & 3 \\
\hline
\end{tabular}

the effects of age, FSIQ, and age and FSIQ) were performed between hippocampal volumes and cognitive measures. Also, Fisher $r$-to- $z$ transformations were applied to assess the significance of the difference between two correlation coefficients (two tailed $p$ value). A $p$-value less than .05 was assumed to denote significant difference.

\section{Results}

There were no significant age differences between groups $(t(26)=-.59, p=.560)$. In addition, non-parametric Mann-Whitney $U$ tests also confirmed that the groups did not differ in socio-economical status $(Z=-.97$, $p=.330)$, but did differ in level of education $(Z=-1.99$, $p=.047$ ) (data is shown in Table 1). No association between gender and group was found $\left(\chi^{2}=.662\right.$, $p=.718)$.

Individuals with WS were found to have significant absolute volume reduction, compared with typically developing participants, in overall white matter $(Z=$ $-3.81, p<.001)$, gray matter $(t(26)=-2.92 p=.007)$ and CSF volumes $(t(26)=-3.84, p=.001)$. Total Intracranial Content (TIC) was also significantly reduced in the
WS group compared with the control group $(t(26)=$ $-4.43, p<.001)$.

Although there was a significant reduction in brain tissue volumes (as can be seen in Table 2), a repeated-measures ANOVA of HF absolute volumes showed no Diagnosis effect $[F(1,26)=.02, p=.880)$. However, a main effect of Side (Left vs Right) $[F(1,26)=10.24$, $p=.004)$ and an interaction between Side and Diagnosis $[F(1,26)=13.79, p=.001)$ was found. There were no significant differences between groups in hippocampal formation volume on either the right $(t(26)=-1.17, p=$ $0.253)$ or left hemisphere $(t(26)=0.86, p=0.400)$ (Fig. 2a). However, when relative volumes of HF were computed (ratio between HF and total gray matter volume), a Diagnosis effect $[F(1,26)=4.34, p=.047)$ a side effect $[F(1,26)=8.12, p=.008)$ and an interaction between Side and Diagnosis $[F(1,18)=11.530, p=.002$ ) were found. Post-hoc t-tests yielded no statistical significant difference between the two groups for the right $\mathrm{HF}$ $(t(26)=1.31, p=.203)$, however the WS group was found to have a significant increase in the volume of left $\operatorname{HF}(t(26)=2.86, p=.008)$ (Fig. 2b). Taking into account the dynamic changes in gray and white matter through adolescence, we analyzed group differences in HF volumes (absolute and adjusted), controlling for the effects of age. Thus, the results remained similar, with no differences between groups in absolute HF volumes on the left $(F(1,26)=.663, p=.443)$ or right hemisphere $(F(1,26)=$ $2.296, p=.143)$ and adjusted right $\operatorname{HF}(F(1,26)=4.083$, $p=.055)$. Again, we observed that WS group was had a significant increase in the volume of adjusted left HF $(F(1,26)=15.267, p=.001)$.

Additionally, when right-left HF asymmetry ratio was analyzed, WS subjects demonstrated a lack of normal asymmetry $(M=.37, \mathrm{SD}=4.53)$, i.e., normal right $>$ left

Table 2 Volumes of TIC, white matter, gray matter, CSF, hippocampal formation volumes (absolute and adjusted to gray matter volume) and asymmetry degree

\begin{tabular}{|c|c|c|c|c|c|c|}
\hline \multirow[t]{2}{*}{ Region and volume type (ml) } & \multicolumn{2}{|c|}{ WS group $(N=15)$} & \multicolumn{2}{|c|}{ Typically developing group $(N=13)$} & \multirow[t]{2}{*}{$t(26)$ or $Z^{\mathrm{a}}$} & \multirow[t]{2}{*}{$p$ value } \\
\hline & $M$ & SD & $M$ & SD & & \\
\hline TIC & 1174.67 & 118.43 & 1402.52 & 148.89 & -4.43 & .001 \\
\hline White matter & 388.16 & 64.87 & 483.68 & 63.17 & -3.81 & .001 \\
\hline Gray matter & 650.20 & 65.82 & 725.39 & 67.57 & -2.92 & .007 \\
\hline CSF & 136.31 & 33.13 & 193.44 & 44.19 & -3.84 & .001 \\
\hline HF-right & 2.92 & .33 & 3.06 & .29 & -1.17 & .25 \\
\hline HF-left & 2.94 & .39 & 2.83 & .20 & 0.86 & .40 \\
\hline Adjusted HF-right $\lambda$ & 4.53 & .62 & 4.28 & .27 & 1.31 & .20 \\
\hline Adjusted HF-left $\lambda$ & 4.55 & .70 & 3.98 & .34 & 2.65 & .01 \\
\hline Asymmetry index & .37 & 4.53 & -7.42 & 6.53 & 3.71 & .001 \\
\hline
\end{tabular}

${ }^{a}$ Two-tailed $t$ tests were performed for relative volume comparisons on TIC, gray matter, CSF, left and right hippocampal formation (absolute and relative) and asymmetry degree, and Mann-Whitney tests were performed for white matter volume; $\lambda \times 10^{3}$ 
(A)

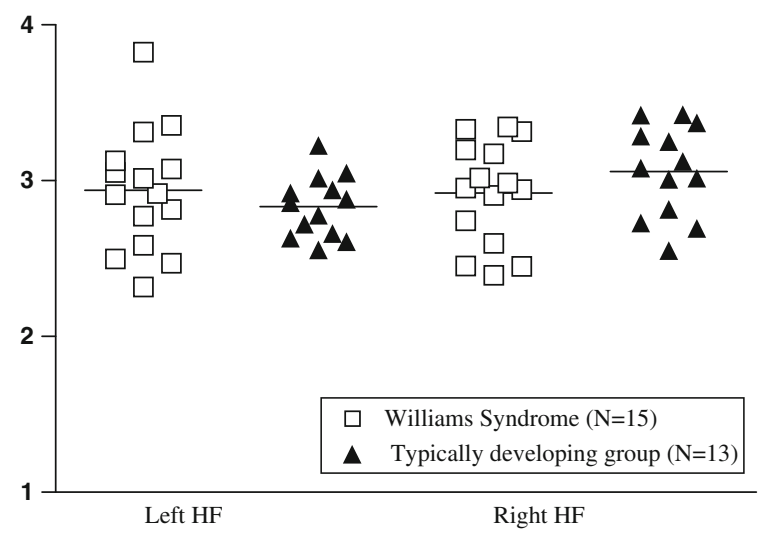

(B)

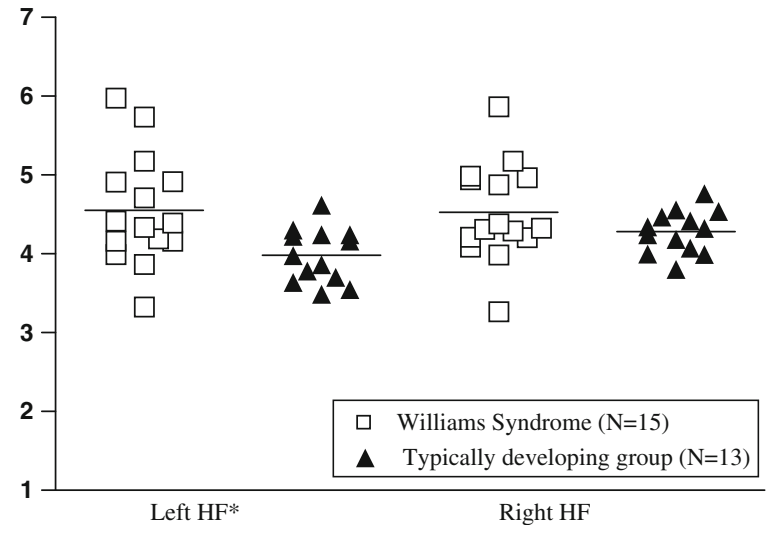

Fig. 2 Scatter plots of left and right absolute (a) and relative (b) HF volumes in WS and in typically developing groups. * Student't $t$ test showed that left HF $(t(26)=2.653, p<.05)$ relative volumes were significantly higher in subjects with WS than normal comparison subjects. Horizontal lines represent means

HF predominance found in the control group $(M=-7.42$, $\mathrm{SD}=6.53) ; t(26)=3.71, p=.001)$.

\section{Memory Performance}

As can be observed in Table 3, individuals with WS showed lower scores in all memory tasks, when compared with the control group. This lower performance was evident in verbal and non-verbal memory tasks, as well as in short and long term memory storage systems.

\section{Correlation of Hippocampal Volume with Memory} Measures

Correlational analysis between memory measures and neuroanatomical measures revealed a significant positive correlation between Left HF absolute volumes and Digit Span $(r=.635, p=.020)$ in the typically developing group, but not in the WS group $(r=-.322, p=.308$; Fisher's $Z$-transformation $p=.011$ ). Also, the asymmetry index was correlated with a visual-spatial memory task (Corsi Blocks) in the control group $\left(\mathrm{r}_{s p}=-.721\right.$, $p=.012)$, but not in the WS group $\left(\mathrm{r}_{s p}=-.500\right.$, $p=.117$; although Fisher's Z-transformation was not significant, $p=.400$ ).

When HF volumes were adjusted for overall gray matter volume (i.e., relative volume of $\mathrm{HF}$ ), we found significant negative correlations with total recalled words in the California Verbal Learning Test with the Right HF $\left(\mathrm{r}_{s p}=\right.$ $-0.689, p=.013)$ and the left $\mathrm{HF} \quad\left(\mathrm{r}_{s p}=-0.678\right.$, $p=.015)$ in the typically developing group while no significant correlations were observed for WS group within the Right HF $\left(\mathrm{r}_{s p}=.429, p=.126\right.$; Fisher's Z-transformation $p=.02)$ and the left $\mathrm{HF}\left(\mathrm{r}_{s p}=.418, p=.137\right.$; Fisher's Z-transformation $p=.003$ ).

Taking into account that FSIQ may influence association between hippocampal measures and cognitive functioning, we performed the same analysis, using partial correlations, controlling for FSIQ effect. Thus, we observed that results were similar for all measures, with exception of the correlation between Digit Span and left HF, that was not found in both typically developing $(r=.569, p=.110)$ and WS group $(r=.208, p=.564)$.

Table 3 Performance in Different Memory Subtests

\begin{tabular}{|c|c|c|c|c|c|}
\hline & \multicolumn{2}{|c|}{ WS group $(N=15)$} & \multicolumn{2}{|c|}{ Typically developing group $(N=13)$} & \multirow[t]{2}{*}{$Z$} \\
\hline & $M$ & SD & $M$ & SD & \\
\hline CVLT-total number of recalled words in 5 trials & 32.93 & 11.03 & 54.92 & 11.88 & $-3.48 * *$ \\
\hline CVLT-long-term memory & 6.42 & 1.93 & 11.85 & 2.19 & $-3.98 * *$ \\
\hline CVLT-recognition & 11.25 & 3.60 & 14.77 & 1.54 & $-2.30^{*}$ \\
\hline Digit span & 8.75 & 2.14 & 16.62 & 2.93 & $-4.27 * *$ \\
\hline Corsi span & 7.09 & 2.26 & 15.64 & 2.11 & $-3.93 * *$ \\
\hline Rey-Osterrieth memory recall & 17.00 & 12.20 & 42.58 & 11.30 & $-3.947 * *$ \\
\hline
\end{tabular}

$* p<.01 ; * * p<.001$ 


\section{Discussion}

The results of this study showed a $16.3 \%$ global reduction in the brain of WS subjects compared with typically developing individuals. However, this reduction of the brain parenchyma was disproportionate, with evidence of absolute volume preservation of HF (left and right), a consistent finding within previous studies (Chiang et al. 2007; Meyer-Lindenberg et al. 2005; Reiss et al. 2004). Moreover, when HF absolute volume was adjusted for overall gray matter volume, an increase in the left HF volume was observed in patients with WS, when compared with typically developing group.

These neuroanatomic data, together with evidence from our cognitive study, revealed that positive correlations between memory measures and HF volumes only occurred in control group. Moreover, a pattern of negative correlations between verbal memory tasks and adjusted HF volumes was found in the typically development group, a finding consistent with previous studies that show this same pattern, specifically when the association between explicit memory measures and adjusted HF volumes is performed (right/brain and left/brain volume ratios) (Chantôme et al. 1999).

Despite the evidence of volume preservation in WS, we suggest that this brain structure might be structurally and functionally impaired in this genetic syndrome, mainly if we take into account the neurocognitive data derived from the current study (impairment in several memory measures in WS) as well as evidence from other studies addressing memory abilities in WS (Brock et al. 2006; Sampaio et al. 2008) and HF integrity in high functioning individuals with WS (Meyer-Lindenberg et al. 2005) and with developmental delay (Chiang et al. 2007; Reiss et al. 2004). Indeed, the hypothesis of structurally impaired HF is consistent with the fact individuals with WS consistently display difficulties in visual-spatial navigation tasks and long-term memory, both in verbal and spatial domains (Brock et al. 2006; O'Hearn et al. 2005; Sampaio et al. 2008; Vicari et al. 2003, 1996). Finally, this possibility is in agreement with the same evidence of enlargement of HF in other neurodevelopmental disorders (Schumann et al. 2004) and with data obtained from knock out mice models with a WS-like genotype, that show volume preservation despite evidence of structural (e.g., defects in neuronal structure like abnormal morphology of dendritic spines) and functional abnormalities (Hoogenraad et al. 2002; Zhao et al. 2005).

Irrespective, however, of the underlying cognitive correlations, the preservation of HF volume in WS subjects is important and illustrates a differential neurodevelopmental pattern, when compared to other brain regions. The HF is a highly plastic region of the brain, where high neuronal turnover persists throughout adult life (Dong et al. 2003; Gould et al. 1999a, 1999b). Indeed, there are indirect observations that brain development in WS is atypical and histopathological abnormalities, like increased neuronal size in cell packing densities in visual and auditory cortices have been described in this syndrome (Galaburda et al. 2002; Holinger et al. 2005). Our findings, which cannot directly assess these issues, are nonetheless consistent with the finding of increased HF in WS in the current study.

The lack of asymmetry in the HF in our WS patients is another relevant finding of this study. Our data suggest that this finding is specific to a disproportional increase of left HF in WS. Taking into account the observation that apoptotic processes are more pronounced in left HF in adult animal models (Silva et al. 2006), disproportional enlargement of left HF in WS might be associated with impairment of apoptotic processes, an important mechanism that shapes brain development.

This lack of asymmetry in HF is also consistent with previous reports of an elevated bilateral symmetry in this clinical group (Sampaio et al. 2008; Van Essen et al. 2006). Despite Chiang et al. (2007) found a trend for a larger right hippocampus in WS with respect to control group, this finding did not reach statistical significance. Also, they did not provide information concerning the lateralization (right or left handed) of the participants (both WS and controls). Indeed, HF is an asymmetric structure, with larger volumes in right with respect to left evidenced in children (Giedd et al. 1996; Utsunomiya et al. 1999) and in adults (Pedraza et al. 2004). However, this asymmetry was not observed in our cohort of WS subjects and a greater right $>$ left asymmetry was positively correlated with performance on a visual-spatial memory task only in the typically development group. Also, left HF was positively correlated with a verbal memory task only in the typically development group (which was associated with FSIQ). These correlations clearly suggest the functional lateralization of $\mathrm{HF}$ according to verbal or visual items (Papanicolaou et al. 2002; Piekema et al. 2006). The lateralization of the HF is likely to be associated with greater complexity and reflect functional specialization, as structural and functional asymmetries are characteristic of biological systems and are associated with lateralization and cognitive skills (Pascual et al. 2004). Importantly, this feature was not present in the WS group, suggesting that, despite HF volume preservation, it is functionally and structurally impaired, possibly associated with abnormal neurodevelopmental abnormalities occurring during embryogenesis as a consequence of the deleted genes. Atypical patterns of structural and functional asymmetries have also been shown in patients with schizophrenia and dyslexia (Edgar et al. 2006; Larsen 1990). Thus, the lack of asymmetry observed in this clinical population is additional evidence 
for structural alterations in HF morphology that might be associated with abnormal brain development and memory impairments. Additionally, disproportional enlargement of HF may underlie impairments in different declarative memory system in WS (Brock et al. 2006; O'Hearn et al. 2005; Sampaio et al. 2008; Vicari et al. 2003, 1996).

The present study provides evidence that HF is structurally abnormal in WS. Proportionally enlarged HF volumes and the loss of the normal right $>$ left asymmetry in WS patients suggest that abnormal neurodevelopmental processes occur in WS subjects, that might correlate with their declarative (verbal and visuo-spatial) memory impairments.

Limitation of this study include the small sample of both WD and typically developing individuals, with a high variable age range (adolescence through adulthood) in which several dynamic events in gray and white matter brain tissues occur, as well as considerable difference in intellectual functioning of both groups under analysis. Thus, future studies should use larger samples, and focus on longitudinal approaches to analyze the development patterns of this brain region, as well analyzing major white matter HF projections to search for abnormal connections and to clarify the functional role of this brain region in WS.

Acknowledgments This research was supported by the grants PIC/ IC/83290/2007 from Fundação para a Ciência e Tecnologia (Portugal). This study was also supported, in part, by grants from the National Institutes of Health (K05 MH 01110).

\section{References}

Bernstein, J. H., \& Waber, D. P. (1996). Developmental scoring system for the Rey-Osterreith complex figure: Professional manual. Odessa, FL: Psychological Assessment Resources, Inc.

Brock, J., Brown, G. D., \& Boucher, J. (2006). Free recall in Williams syndrome: Is there a dissociation between short- and long-term memory? Cortex, 42(3), 366-375.

Carr, V. A., Viskontas, I. V., Engel, S. A., \& Knowlton, B. (in press). Neural activity in the hippocampus and perirhinal cortex during encoding is associated with the durability of episodic memory. Journal of Cognitive Neuroscience.

Casasanto, D. J., Killgore, W. D., Maldjian, J. A., Glosser, G., Alsop, D. C., Cooke, A. M., et al. (2002). Neural correlates of successful and unsuccessful verbal memory encoding. Brain and Language, 80(3), 287-295.

Chantôme, M., Perruchet, P., Hasboun, D., Dormont, D., Sahel, M., Sourour, N., et al. (1999). Is there a negative correlation between explicit memory and hippocampal volume? NeuroImage, 10, 589-595.

Chiang, M. C., Reiss, A. L., Lee, A. D., Bellugi, U., Galaburda, A. M., Korenberg, J. R., et al. (2007). 3D pattern of brain abnormalities in Williams syndrome visualized using tensorbased morphometry. Neuroimage, 36(4), 1096-1109.

Corsi, P. M. (1972). Human memory and the medial temporal region of the brain. Unpublished doctoral dissertation, McGill University.
Delis, D., Kramer, J. H., Kaplan, E., \& Ober, B. A. (1994). California verbal learning test-children's version: Manual. San Antonio: The Psychological Corporation.

Delis, D., Kramer, J. H., Kaplan, E., \& Ober, B. A. (2000). California verbal learning test-second edition, adult version-manual. San Antonio: The Psychological Corporation.

Dong, H., Csernansky, C. A., Goico, B., \& Csernansky, J. G. (2003). Hippocampal neurogenesis follows kainic acid-induced apoptosis in neonatal rats. Journal of Neuroscience, 23(5), 1742-1749.

Duvernoy, H. M. (2005). The human hippocampus. functional anatomy, vascularization and serial sections with MRI (3rd ed.). Berlin: Springer-Verlag Berlin Heidelberg.

Edgar, J. C., Yeo, R. A., Gangestad, S. W., Blake, M. B., Davis, J. T., Lewine, J. D., et al. (2006). Reduced auditory M100 asymmetry in schizophrenia and dyslexia: Applying a developmental instability approach to assess atypical brain asymmetry. Neuropsychologia, 44(2), 289-299.

Eichenbaum, H., Dudchenko, P., Wood, E., Shapiro, M., \& Tanila, H. (1999). The hippocampus, memory, and place cells: Is it spatial memory or a memory space? Neuron, 23(2), 209-226.

Fernandez, G., Brewer, J. B., Zhao, Z., Glover, G. H., \& Gabrieli, J. D. (1999). Level of sustained entorhinal activity at study correlates with subsequent cued-recall performance: A functional magnetic resonance imaging study with high acquisition rate. Hippocampus, 9(1), 35-44.

Galaburda, A. M., Holinger, D. P., Bellugi, U., \& Sherman, G. F. (2002). Williams syndrome: Neuronal size and neuronal-packing density in primary visual cortex. Archives of Neurology, 59(9), 1461-1467.

Gazzaley, A., Rissman, J., \& Desposito, M. (2004). Functional connectivity during working memory maintenance. Cognitive, Affective and Behavioral Neuroscience, 4(4), 580-599.

Giedd, J. N., Vaituzis, A. C., Hamburger, S. D., Lange, N., Rajapakse, J. C., Kaysen, D., et al. (1996). Quantitative MRI of the temporal lobe, amygdala, and hippocampus in normal human development: Ages 4-18 years. Journal of Comparative Neurology, 366(2), 223-230.

Gould, E., Beylin, A., Tanapat, P., Reeves, A., \& Shors, T. J. (1999a). Learning enhances adult neurogenesis in the hippocampal formation. Nature Neuroscience, 2(3), 260-265.

Gould, E., Tanapat, P., Hastings, N. B., \& Shors, T. J. (1999b). Neurogenesis in adulthood: A possible role in learning. Trends in Cognitive Sciences, 3(5), 186-192.

Holinger, D. P., Bellugi, U., Mills, D. L., Korenberg, J. R., Reiss, A. L., Sherman, G. F., et al. (2005). Relative sparing of primary auditory cortex in Williams Syndrome. Brain Research, 1037(12), 35-42.

Hoogenraad, C. C., Koekkoek, B., Akhmanova, A., Krugers, H., Dortland, B., Miedema, M., et al. (2002). Targeted mutation of Cyln2 in the Williams syndrome critical region links CLIP-115 haploinsufficiency to neurodevelopmental abnormalities in mice. Nature Genetics, 32(1), 116-127.

Karlsgodt, K. H., Shirinyan, D., van Erp, T. G., Cohen, M. S., \& Cannon, T. D. (2005). Hippocampal activations during encoding and retrieval in a verbal working memory paradigm. Neuroimage, 25(4), 1224-1231.

Kato, T., Erhard, P., Takayama, Y., Strupp, J., Le, T. H., Ogawa, S., et al. (1998). Human hippocampal long-term sustained response during word memory processing. Neuroreport, 9(6), 1041-1047.

Korenberg, J. R., Chen, X. N., Hirota, H., Lai, Z., Bellugi, U., Burian, D., et al. (2000). VI. Genome structure and cognitive map of Williams syndrome. Journal of Cognitive Neuroscience, 12(1), 89-107.

Larsen, J. P., Hoien, T., Lundberg, I., \& Odegaard, H. (1990). MRI evaluation of the size and symmetry of the planum temporale in 
adolescents with developmental dyslexia. Brain and Language, 39(2), 289-301.

Maguire, E. A., Gadian, D. G., Johnsrude, I. S., Good, C. D., Ashburner, J., Frackowiak, R. S., et al. (2000). Navigationrelated structural change in the hippocampi of taxi drivers. Proceedings of the National Academy of Sciences-USA, 97(8), 4398-4403.

Meyer-Lindenberg, A., Mervis, C. B., Sarpal, D., Koch, P., Steele, S., Kohn, P., et al. (2005). Functional, structural, and metabolic abnormalities of the hippocampal formation in Williams syndrome. Journal of Clinical Investigation, 115(7), 1888-1895.

O'Hearn, K., Landau, B., \& Hoffman, J. E. (2005). Multiple object tracking in people with Williams syndrome and in normally developing children. Psychological Science, 16, 905-912.

Pantel, J., O’Leary, D. S., Cretsinger, K., Bockholt, H. J., Keefe, H., Magnotta, V. A., et al. (2000). A new method for the in vivo volumetric measurement of the human hippocampus with high neuroanatomical accuracy. Hippocampus, 10(6), 752-758.

Papanicolaou, A. C., Simos, P. G., Castillo, E. M., Breier, J. I., Katz, J. S., \& Wright, A. A. (2002). The hippocampus and memory of verbal and pictorial material. Learning and Memory, 9(3), 99-104.

Pascual, A., Huang, K. L., Neveu, J., \& Preat, T. (2004). Neuroanatomy: Brain asymmetry and long-term memory. Nature, 427(6975), 605-606.

Pedraza, O., Bowers, D., \& Gilmore, R. (2004). Asymmetry of the hippocampus and amygdala in MRI volumetric measurements of normal adults. Journal of International Neuropsychology Society, 10(5), 664-678.

Piekema, C., Kessels, R. P., Mars, R. B., Petersson, K. M., \& Fernandez, G. (2006). The right hippocampus participates in short-term memory maintenance of object-location associations. Neuroimage, 33(1), 374-382.

Pinter, J. D., Eliez, S., Schmitt, J. E., Capone, G. T., \& Reiss, A. L. (2001). Neuroanatomy of Down's syndrome: a high-resolution MRI study. American Journal of Psychiatry, 158(10), 16591665.

Pohl, K., Bouix, S., \& Kikinis, R. (2004). Anatomical guided segmentation with nonstationary tissue class distributions in an expectation-maximization framework. IEEE International Symposium on Biomedical Imaging, 81-84.

Pruessner, J. C., Li, L. M., Serles, W., Pruessner, M., Colline, D. L., Kabani, N., et al. (2000). Volumetry of hippocampus and amygdala with high-resolution mri and three-dimensional anslysis software: Minimizing the discrepancies between laboratories. Cerebral Cortex, 10(1047-3211), 433-442.

Ranganath, C., Cohen, M. X., \& Brozinsky, C. J. (2005). Working memory maintenance contributes to long-term memory formation: Neural and behavioral evidence. Journal of Cognitive Neuroscience, 17(7), 994-1010.

Ranganath, C., \& D'Esposito, M. (2001). Medial temporal lobe activity associated with active maintenance of novel information. Neuron, 31(5), 865-873.

Reiss, A. L., Eckert, M. A., Rose, F. E., Karchemskiy, A., Kesler, S., Chang, M., et al. (2004). An experiment of nature: Brain anatomy parallels cognition and behavior in Williams syndrome. Journal of Neuroscience, 24(21), 5009-5015.

Rey, A. (1959). Manuel du test de copie d'une figure complexe de A. Rey. Paris: Les Editions du Centre de Psychologie Appliquée.

Sampaio, A., Sousa, N., Fernandez, M., Henriques, M., \& Goncalves, O. F. (2008a). Memory abilities in Williams syndrome: Dissociation or developmental delay hypothesis? Brain and Cognition, 66(3), 290-297.

Sampaio, A., Sousa, N., Fernandez, M., Vasconcelos, C., Shenton, M. E., \& Goncalves, O. F. (2008b). MRI assessment of superior temporal gyrus in Williams syndrome. Cogenital Behaviour Neurology, 21(3), 150-156.

Schumann, C. M., Hamstra, J., Goodlin-Jones, B. L., Lotspeich, L. J., Kwon, H., Buonocore, M. H., et al. (2004). The amygdala is enlarged in children but not adolescents with autism; the hippocampus is enlarged at all ages. Journal of Neuroscience, 24(28), 6392-6401.

Silva, R., Lu, J., Wu, Y., Martins, L., Almeida, O. F., \& Sousa, N. (2006). Mapping cellular gains and losses in the postnatal dentate gyrus: Implications for psychiatric disorders. Experimental Neurology, 200(2), 321-331.

Utsunomiya, H., Takano, K., Okazaki, M., \& Mitsudome, A. (1999). Development of the temporal lobe in infants and children: Analysis by MR-based volumetry. AJNR. American Journal of Neuroradiology, 20(4), 717-723.

Van Essen, D. C., Dierker, D., Snyder, A. Z., Raichle, M. E., Reiss, A. L., \& Korenberg, J. (2006). Symmetry of cortical folding abnormalities in Williams syndrome revealed by surface-based analyses. Journal of Neuroscience, 26(20), 5470-5483.

Vicari, S., Bellucci, S., \& Carlesimo, G. A. (2003). Visual and spatial working memory dissociation: Evidence from Williams syndrome. Development Medicine and Child Neurology, 45(4), 269-273.

Vicari, S., Brizzolara, D., Carlesimo, G. A., Pezzini, G., \& Volterra, V. (1996). Memory abilities in children with Williams syndrome. Cortex, 32(3), 503-514.

Zhao, C., Aviles, C., Abel, R. A., Almli, C. R., McQuillen, P., \& Pleasure, S. J. (2005). Hippocampal and visuospatial learning defects in mice with a deletion of frizzled 9, a gene in the Williams syndrome deletion interval. Development, 132(12), 2917-2927. 